\title{
PROCESSO DE CONVERSÃO DE GLICEROL EM ÁCIDOS ORGÂNICOS SOB CATALISADOR DE Pd-Bi/C
}

\author{
A. C. CABRAL de LiMA", J. E. B. NETO, N. M. LiMA FILHO, L. C. A. MARANHÃO e C. A. M. \\ ABREU \\ ${ }^{1}$ Universidade Federal de Pernambuco, Departamento de Engenharia Química \\ E-mail para contato: anacscbr@yahoo.com.br
}

\begin{abstract}
RESUMO - Atualmente, a conversão de glicerol tem atraído atenção pelo seu grande potencial de sustentabilidade e nível de funcionalização. Seu uso como matéria-prima em operações da química fina (oxidação, hidrogenólise, etc.) está em destaque por gerar produtos de alto valor agregado. Neste trabalho, estudou-se o processo catalítico de oxidação do glicerol em reator de leito de lama (1L), obtendo-se a cinética de reação, incluindo adsorção/dessorção e etapas de reação na superfície do catalisador e o próximo passo é elevar a escala do processo, com uso de um reator de leito gotejante. As condições foram: $70^{\circ} \mathrm{C}$ a $90^{\circ} \mathrm{C}, 550 \mathrm{rpm}$, razão $\mathrm{NaOH} /$ glicerol de 2 a $4 \mathrm{~mol} / \mathrm{mol}$ e razão glicerol $/ \mathrm{Pd}$ de 3191 e $5319 \mathrm{~mol} / \mathrm{mol}$. Como resultados, obteve-se maior conversão de glicerol $(65 \%)$ para maior temperatura e menor razão de glicerol/Pd, dados o baixo teor de paládio $(\operatorname{Pd}(0,10 \%)-\mathrm{Bi}(0,68 \%) / \mathrm{C})$ e a elevada área superficial $\left(437 \mathrm{~m}^{2} / \mathrm{g}, 0,21 \mathrm{~cm}^{3} / \mathrm{g}\right)$, fornecendo boa seletividade para a produção de ácidos orgânicos.
\end{abstract}

\section{INTRODUÇÃO}

O glicerol tem sido grandemente utilizado por ser considerado como reserva bio-sustentável e pelo seu alto nível de funcionalização (Demirel-Gülen et al., 2005). A conversão catalítica de glicerol em produtos químicos de alto valor agregado via reações de oxidação, redução, halogenação, dehidrogenação, hidrogenólise, pirólise, transesterificação e eterificação, tem chamado a atenção de muitos pesquisadores, devido à elevada produção de glicerol através da reação de transesterificação de ácidos gordos em presença de álcool (etanol). (Sobczac et al., 2010). Portanto, a possibilidade de se obter $100 \mathrm{~kg}$ de glicerol por tonelada de biodiesel (10\% do total da produção) e a dificuldade em purificar o glicerol cru para aplicações nas indústrias de cosméticos, farmacêuticas e de alimentos são alguns dos fatores que têm favorecido a busca por rotas alternativas de produção de intermediários químicos, utilizando o glicerol como matéria-prima.

Especialmente, a oxidação catalítica em fase líquida é uma rota promissora para converter glicerol em compostos úteis, com uso de um catalisador suficientemente ativo e seletivo para a formação de químicos como o ácido glicérico (GCA) e/ou dihidroxiacetona (DHA), potencialmente usados como intermediários químicos na indústria de química fina, particularmente na farmacêutica, de cosméticos, em síntese orgânica e como suplemento nutricional (Hu et al., 2011). A Figura 1 representa o mecanismo de reação para a oxidação de glicerol em presença do catalisador de $\mathrm{Pd}-\mathrm{Bi} / \mathrm{C}$. 


\section{9 a 22 de outubro de 2014 \\ Florianópolis/SC}

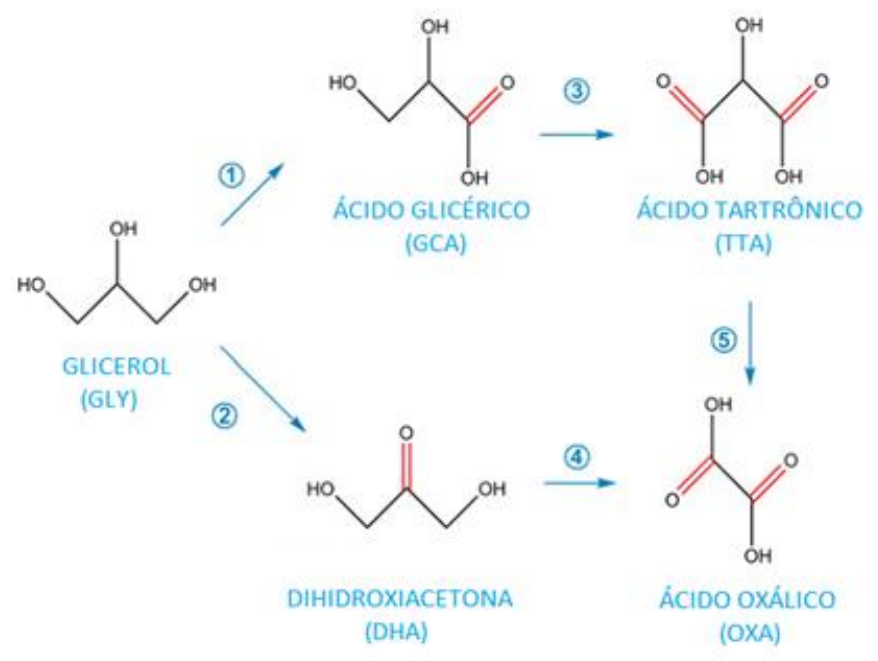

Figura 1 - Esquema reacional de oxidação catalítica do glicerol.

Entretanto, o processo de oxidação do glicerol pode ser bastante complexo do ponto de vista da separação de produtos. Porém, isto pode ser melhorado com o controle da seletividade da reação, a partir da combinação de fatores como a escolha adequada do metal, do tamanho da partícula e do suporte, bem como das condições de operação como pressão, temperatura e quantidade de base e metal (Fordham et al., 1996; Demirel-Gülen et al., 2005). Ambos, o catalisador e as condições de operação têm uma influência significativa na seletividade dos produtos e isso determina a estabilidade dos intermediários e sua subsequente conversão. Para a oxidação do glicerol, os metais Pd e Pt têm sido extensivamente usados como catalisadores ativos, mas eles têm significativa desvantagem pelo envenenamento por oxigênio, que é proporcional à pressão deste, o que leva à desativação, segundo Prati e Porta (2004). Já o carvão ativado, apresenta-se como melhor suporte catalítico que os óxidos, devido às suas propriedades específicas, como maior resistência a meios ácidos e alcalinos, sua porosidade e superfície química controlada e pela possibilidade de se recuperar o metal por combustão do suporte (Serp et al., 2003; Rodríguez-Reinoso, 1998).

Neste trabalho, optou-se por um catalisador com baixos teores de Pd e Bi, suportados em carvão ativado, uso do ar comprimido como fonte de oxigênio, condições de temperatura mais severas e

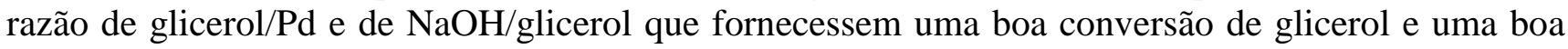
seletividade, principalmente, para ácido glicérico e dihidroxiacetona.

\section{EXPERIMENTAL}

As operações de oxidação do glicerol ocorreram em reator de leito de lama (1L - Figura 2), acoplado a um banho ultra termostático para aquecimento do reator, sistema automático de agitação e medição de temperatura. As condições foram as seguintes: concentração inicial de glicerol de $0,3 \mathrm{M}$ $(0,5 \mathrm{~L})$, temperaturas de $70^{\circ} \mathrm{C}$ a $90^{\circ} \mathrm{C}$, agitação de $550 \mathrm{rpm}$, razão $\mathrm{NaOH} / \mathrm{glicerol} \mathrm{de} 2$ e $4 \mathrm{~mol} / \mathrm{mol} \mathrm{e}$ 
razão glicerol/Pd de 3191 e $5319 \mathrm{~mol} / \mathrm{mol}$.

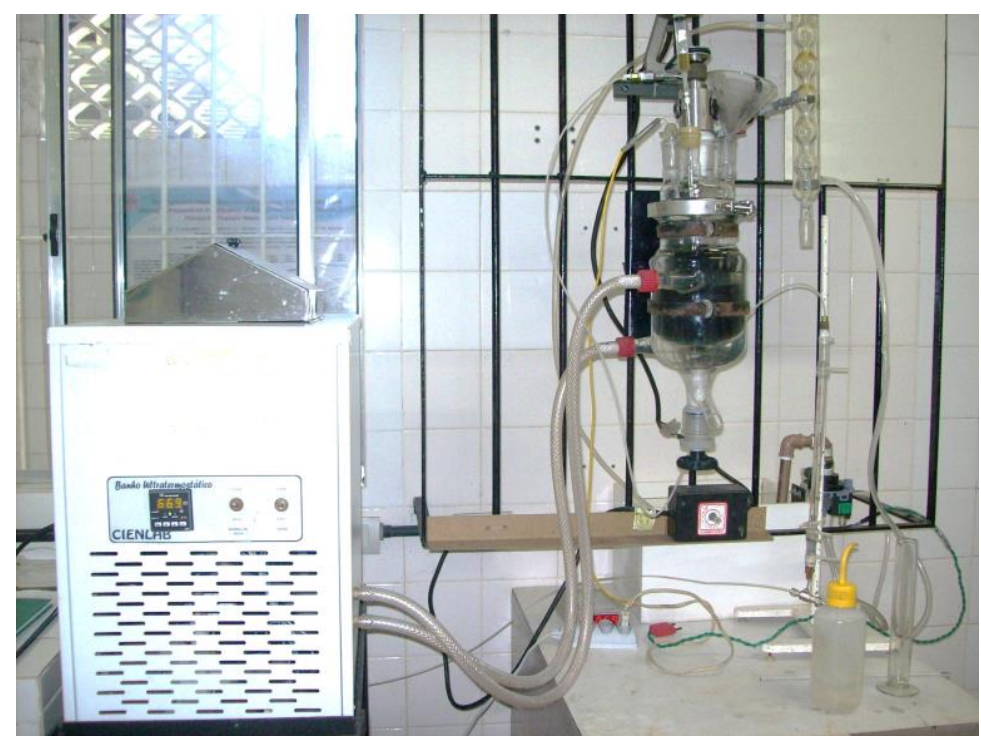

Figura 2 - Reator de mistura encamisado (direita) para reações de oxidação e banho ultratermostático (esquerda).

O catalisador foi preparado por impregnação úmida sucessiva de soluções de $\mathrm{PdCl}_{2}$ e $\mathrm{Bi}\left(\mathrm{NO}_{3}\right) 3$ ao suporte, fornecido pela Carbomafra. Utilizou-se um rota-evaporador por $6 \mathrm{~h}$ a $60^{\circ} \mathrm{C}$ sob agitação, seguido de secagem a $100^{\circ} \mathrm{C}$ por $24 \mathrm{~h}$, redução em presença de hidrogênio na vazão de $0,075 \mathrm{~L}^{-\mathrm{h}^{-1}} \mathrm{~g}_{\text {cat }}{ }^{-}$ ${ }^{1}$, temperatura de $260^{\circ} \mathrm{C}$, com rampa de aquecimento de $10{ }^{\circ} \mathrm{C} \cdot \mathrm{min}^{-1}$, por $3 \mathrm{~h}$. A caracterização foi realizada por análise química, usando um espectrômetro de fluorescência de raios-X (Rigaku modelo RIX 3000), equipado com tubo de $\mathrm{Rh}$. A análise de difração de raios-X foi realizada num difratômetro de raios-X modelo D-5000 (Siemens), sob radiação de $\mathrm{Cu}-\mathrm{K} \alpha$, sendo as amostras escaneadas sob ângulo de Bragg (20) na faixa de 10 - 90 . A microscopia eletrônica de varredura (MEV) foi realizada em JEOL JSM-5900 (Scanning Electron Microscope / Noran instruments), admitindo-se 10kV; magnificação de x100; distância de trabalho de 16-17 mm; $100 \mu$ m e spotsize 42. As curvas de adsorção de acordo com o método de Brunauer-Emmett-Teller (BET), em aparelho ASAP 2010.

As amostras de reação foram coletadas, em intervalos de 30 a 60 min e analisadas off-line em um Cromatógrafo Líquido de Alta Eficiência (CLAE - Varian Prostar), utilizando coluna Aminex $\mathrm{HPX} 87 \mathrm{H}$ a $50^{\circ} \mathrm{C}$, fase móvel de $\mathrm{H}_{2} \mathrm{SO}_{4}(5 \mathrm{mM})$, a $0,6 \mathrm{~mL} / \mathrm{min}$.

A cinética proposta para a reação de oxidação do glicerol inclui etapas de adsorção/dessorção e de reação na superfície do catalisador, segundo um modelo de Langmuir-Hinshelwood, conforme a Equação 1, admitindo-se sítios ativos distintos para o oxigênio e para o glicerol (Neto e Lima Filho, 2009). 
$\mathrm{r}_{\mathrm{i}}=\frac{k_{i j}^{\prime} C_{j}}{\left(1+K_{G L Y} C_{G L Y}\right)}$

Sendo $k_{i j}^{\prime}$ uma pseudo-constante cinética dada por:

$k_{i j}^{\prime}=\frac{k_{i} K_{j} K_{O_{2}} C_{O_{2}}}{\left(1+K_{O_{2}} C_{O_{2}}\right)}$

onde $\mathrm{i}=(1,2,3,4$ e 5) de acordo com o sentido de reação. Enquanto, j = (GLY, OXA, TTA, GCA e DHA). $k_{i}$ é constante cinética e $K_{j}$ e $K_{O 2}$, são as constantes de equilíbrio de adsorção para os componentes e o oxigênio, respectivamente.

\section{RESULTADOS}

A caracterização do catalisador, por fluorescência de raios-X mostrou que foi impregnado ao suporte o equivalente a $0,10 \% \mathrm{Pd}$ e $0,68 \% \mathrm{Bi}$. A Tabela 1 apresenta os dados de BET do carvão e do catalisador, indicando elevada área superficial, mesmo após a impregnação do paládio. As Figuras 3 e 4 apresentam o difratrograma de raios-X e o MEV do catalisador de $\mathrm{Pd}-\mathrm{Bi} / \mathrm{C}$.

Tabela 1 - BET do catalisador de Pd-Bi/C.

\begin{tabular}{|l|c|c|}
\hline Amostras & C & Pd-Bi/C \\
\hline $\mathrm{S}_{\mathrm{BET}}\left(\mathrm{m}^{2} / \mathrm{g}\right)$ & 660,00 & 436,90 \\
\hline $\mathrm{Vp}\left(\mathrm{cm}^{3} / \mathrm{g}\right)$ & 0,32 & 0,21 \\
\hline $\mathrm{d}_{\mathrm{p}}(\AA)$ & 19,40 & 19,14 \\
\hline
\end{tabular}

O caráter amorfo do carvão ativado foi mais evidente que os picos referentes ao $\mathrm{Pd}$ e ao $\mathrm{Bi}$, devido à baixa concentração desses metais inserida no suporte. Por essa razão também, ficando abaixo do limite de detecção do difratrômetro. Entretanto, mesmo estando em baixa concentração, os picos do paládio na forma metálica podem ser sugeridos no ângulo de $40,02^{\circ}$ e $46,24^{\circ}$, conforme citado por Hammoudeh e Mahmoud (2003) e de acordo com Belkacemi e Hamoudi (2010), em ângulos de $45^{\circ}$ e $82^{\circ}$ e, além disso, um pico adicional a aproximadamente $38^{\circ}$ pode representar a formação de um composto de natureza intermetálica, o $\mathrm{Bi}_{\mathrm{x}} \mathrm{Pd}_{\mathrm{y}}$. 


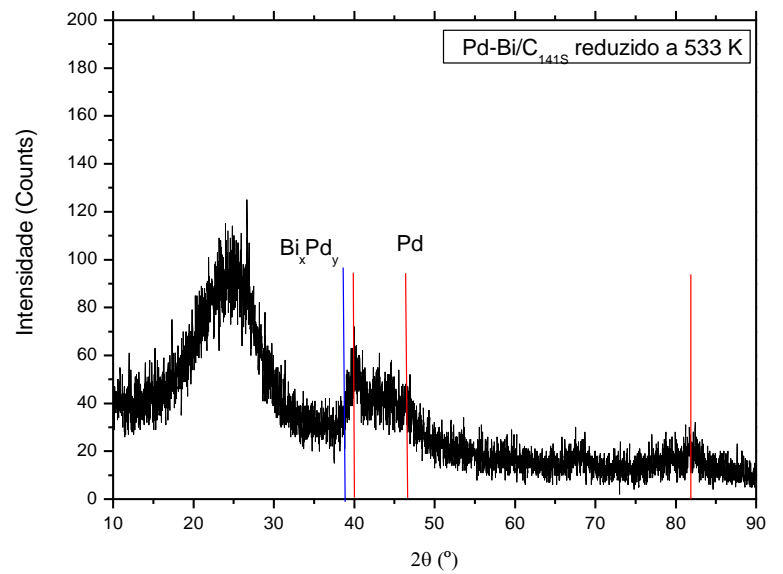

Figura 3 - Difratograma de raios-X do catalisador de $\mathrm{Pd}-\mathrm{Bi} / \mathrm{C}$, reduzido a $260^{\circ} \mathrm{C}$. Condições: escaneamento sob ângulo de Bragg (20) na faixa de $10-90^{\circ}$.

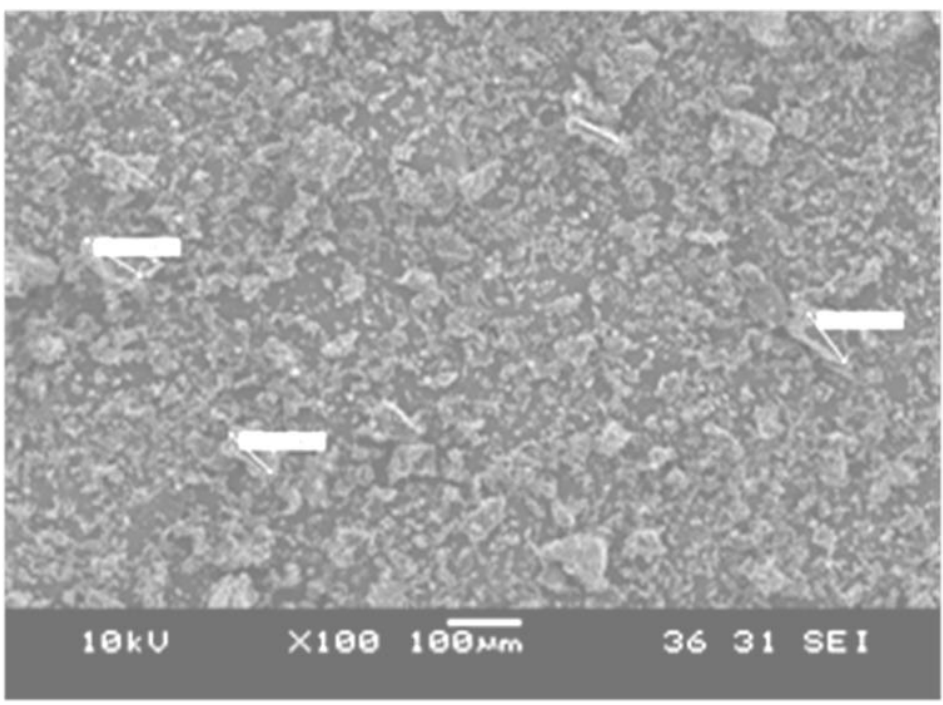

Figura 4 - MEV do catalisador $\mathrm{Pd}-\mathrm{Bi} / \mathrm{C}_{141 \mathrm{~s}}$ reduzido a $260^{\circ} \mathrm{C}$.

$\mathrm{O}$ resultado do $\mathrm{MEV}$ do catalisador de $\mathrm{Pd}-\mathrm{Bi} / \mathrm{C}$ demonstrou a morfologia irregular do suporte de carvão ativado, mas também as superfícies referentes aos materiais porosos estudados e o aparente espalhamento dos metais nas superfícies do suporte. 
Com relação à oxidação do glicerol foi possível obter os perfis de concentração de glicerol e dos produtos, levando-se em consideração a concentração inicial do glicerol, as temperaturas e as razões de $\mathrm{NaOH} /$ glicerol e de glicerol/Pd. A Figura 5 apresenta (a) Efeito da temperatura na oxidação de glicerol a $90^{\circ} \mathrm{C}$, (b) Conversão de glicerol nas três temperaturas, (c) Seletividade para ácido glicérico e (d) Seletividade para dihidroxiacetona, respectivamente. A Figura 6 apresenta (a) Efeito da razão de $\mathrm{NaOH} / \mathrm{GLY}$ no consumo de glicerol (b) Efeito da razão GLY/Pd na produção de GCA e DHA, respectivamente.

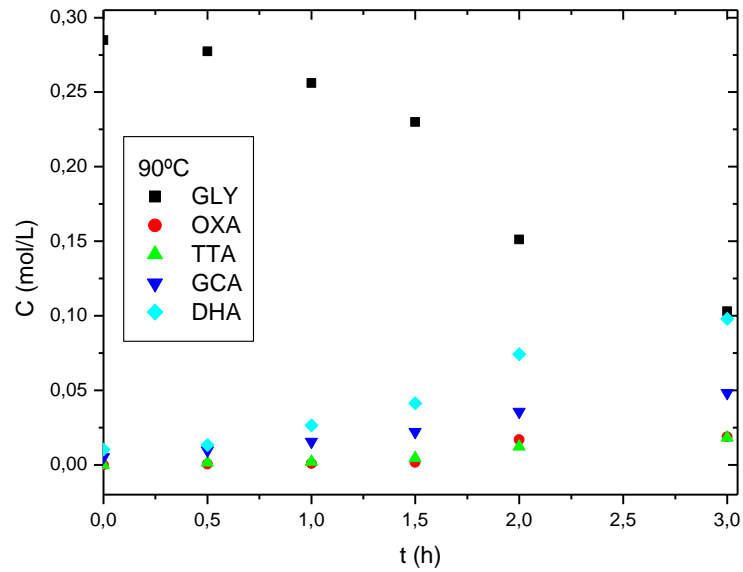

(a)

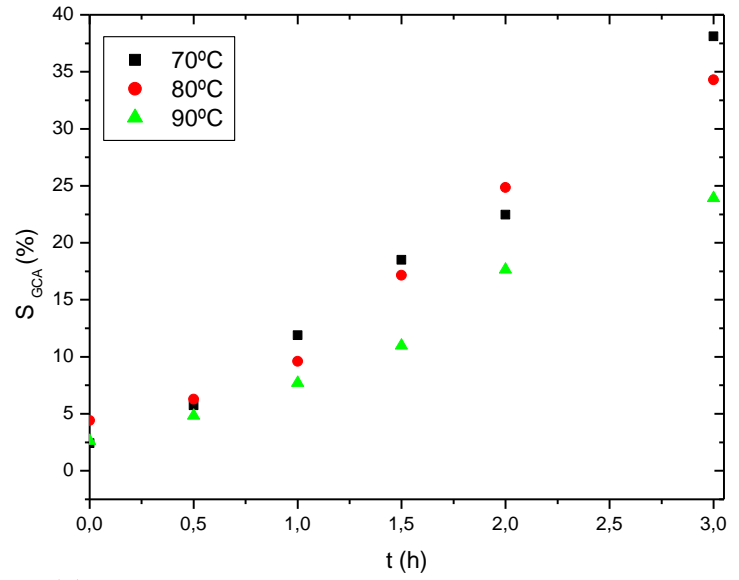

(c)

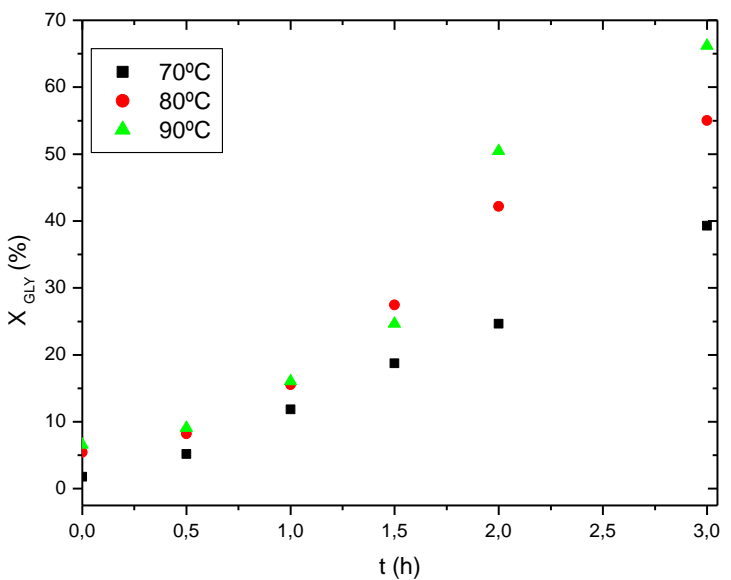

(b)

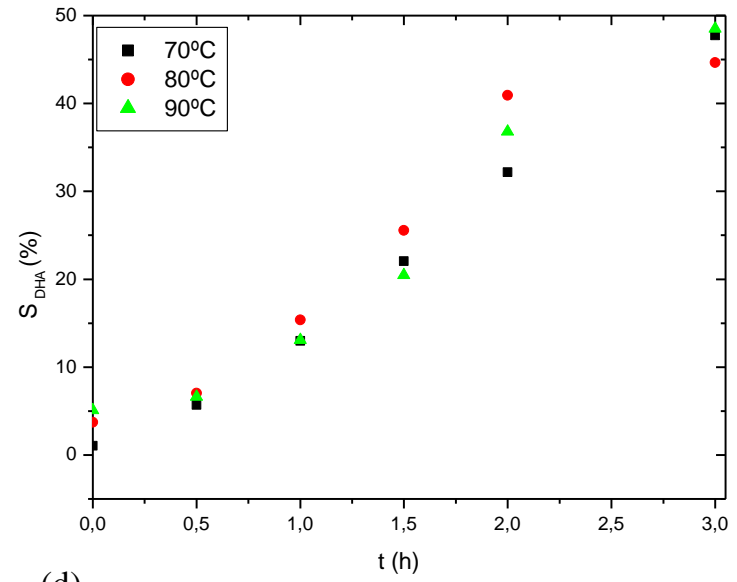

(d)

Figura 5 - (a) Efeito da temperatura na oxidação do glicerol; (b) Conversão de glicerol; (c) Seletividade para Ácido glicérico; (c) Seletividade para Dihidroxiacetona. Condições: $\mathrm{C}_{0, \mathrm{GLY}}=0,3 \mathrm{M}$, agitação $550 \mathrm{rpm}, \mathrm{T}=70-90^{\circ} \mathrm{C}, \mathrm{P}_{\mathrm{O} 2}=1 \mathrm{bar}, \mathrm{NaOH} / \mathrm{GLY}=4 \mathrm{~mol} / \mathrm{mol}, \mathrm{GLY} / \mathrm{Pd}=3191 \mathrm{~mol} / \mathrm{mol}$. 


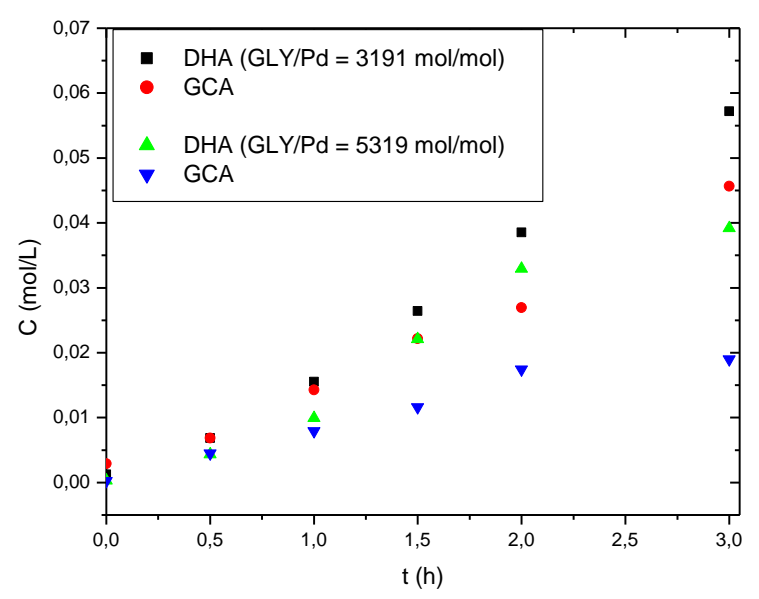

(a)

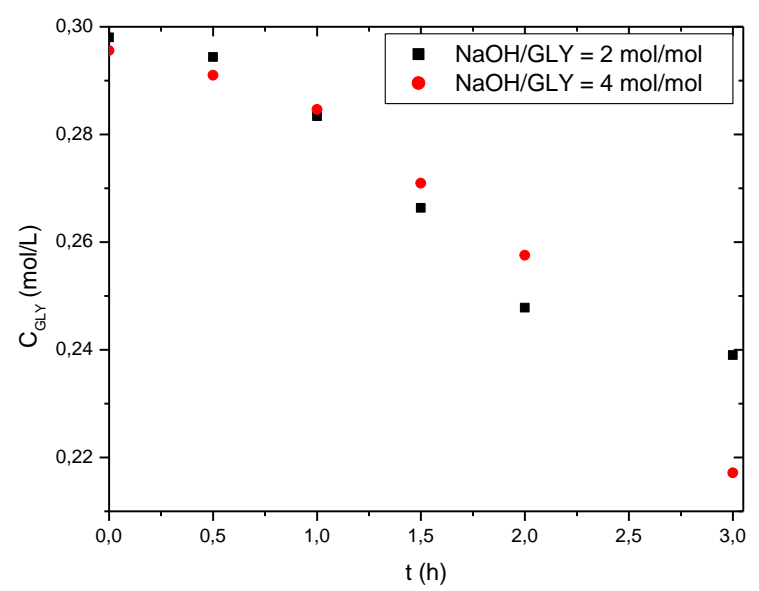

(b)

Figura 6 - (a) Efeito da razão GLY/Pd na produção de GCA e DHA; (b) Efeito da razão de $\mathrm{NaOH} / \mathrm{GLY}$ no consumo de glicerol. Condições: $\mathrm{C}_{0, \mathrm{GLY}}=0,3 \mathrm{M}, \mathrm{T}=70^{\circ} \mathrm{C}, \mathrm{P}_{\mathrm{O} 2}=1$ bar, agitação 550 $\mathrm{rpm}, \mathrm{GLY} / \mathrm{Pd}=3191 \mathrm{~mol} / \mathrm{mol}$.

De acordo com os resultados, o aumento na temperatura de reação, utilizando o catalisador de $\operatorname{Pd}(0,10 \%)-\mathrm{Bi}(0,68 \%) / \mathrm{C}$, nas condições de operação selecionadas, favorece a conversão do glicerol, aumenta a produção de DHA e, consequentemente, de OXA. A $70^{\circ} \mathrm{C}$, a conversão de glicerol foi de $40 \%$, enquanto que a $90^{\circ} \mathrm{C}$ chegou a $65 \%$, comprovando o efeito da temperatura na atividade do catalisador. Na menor razão de GLY/Pd foi possível obter maior consumo de glicerol e maior seletividade para DHA e GCA. Já a influência da razão molar de NaOH/GLY foi melhorada ao passar de 2 para $4 \mathrm{~mol} / \mathrm{mol}$, admitindo-se que a primeira etapa do mecanismo de reação envolve dehidrogenação do grupo álcool do glicerol pelo grupo hidroxila da base e isto é seguido pela formação de intermediários de oxidação. Então, a conversão do glicerol tem grande dependência da quantidade de base no meio, segundo (Sobczak et al., 2009; Carrettin et al., 2003; Ketchie et al., 2007; Dimitratos et al., 2009). Por fim, observou-se em todos os experimentos que a seletividade para DHA sobressai em relação ao GCA, fato que está relacionado ao meio alcalino, à estabilidade e seletividade do catalisador.

\section{REFERÊNCIAS}

BELKACEMI, K.; HAMOUDI, S. Chemocatalytic oxidation of lactose to lactobionic acid over PdBi/SBA-15: reaction kinetics and modeling. Ind. Eng. Chem. Res., v. 19, p. 6878 - 6889, 2010.

CARRETTIN, S.; MCMORN, P.; JOHNSTON, P.; GRIFFIN, K.; HUTCHINGS, G. J.; KIELLY, C. J. Oxidation of glycerol using supported Pt, Pd and Au catalysts. Phys. Chem., v. 5, p. 1329-1336, 2003.

DEMIREL-GÜLEN, S.; LUCAS, M.; CLAUS, P. Liquid phase oxidation of glycerol over carbon 
supported gold catalysts. Catal. Today, v. 102, p. 166-172, 2005.

DIMITRATOS, N.; VILLA, A.; PRATI, L. Liquid phase oxidation of glycerol using a single phase $(\mathrm{Au}-\mathrm{Pd})$ alloy supported on activated carbon: effect of reaction conditions. Catal. Lett., v. 133, p. 334-340, 2009.

FORDHAM, P.; GARCIA, R.; BESSON, M.; GALLEZOT, P. Selective catalytic oxidation with air of glycerol and oxygenated derivatives on platinum metals. Stud. Surf. Sci. Catal. v. 101, p. 161170, 1996.

HAMMOUDEH, A.; MAHMOUD, S. Selective hydrogenation of cinnamaldehyde over $\mathrm{Pd} / \mathrm{SiO}_{2}$ catalysts: selectivity promotion by alloyed Sn. Journal of Molecular Catalysis A: Chemical, v. 203, p. 231-239, 2003.

HU, W.; LOWRY, B.; VARMA, A. Kinetic study of glycerol oxidation network over Pt-Bi/C catalyst. Applied Catalysis B:Environmental, v. 106, p. 123- 132, 2011.

KETCHIE, W.C.; MURAYAMA, M.; DAVIS, R.J. Promotional effect of hydroxyl on the aqueous phase oxidation of carbon monoxide and glycerol over supported Au catalysts. Top. Catal., v. 44 p. 307-317, 2007.

NETO, J. E. B.; LIMA FILHO, N. M. Valorização catalítica de glicerina residual do biodiesel: para produção de ácidos orgânicos. XVII Congresso de Iniciação Científica e I Congresso de Iniciação em Desenvolvimento Tecnológico e Inovação. 2009.

PRATI, L.; PORTA, F. Selective oxidation of glycerol to sodium glycerate with gold on carbon catalyst: an insight into reaction selectivity. J. Catal., v. 224, p. 397-403, 2004.

RODRÍGUEZ-REINOSO, F. The role of carbon materials in heterogeneous catalysis. Carbon, v. 36, 159-175, 1998.

SERP, P.; CORRIAS, M.; KALCK, P. Carbon nanotubes and nanofibers in catalysis. Appl. Catal., v. A 253, p. 337-358, 2003.

SOBCZAK, I.; JAGODZINSKA, K.; ZIOLEK, M. Glycerol oxidation on gold catalysts supported on group five metal oxides - a comparative study with other metal oxides and carbon based catalysts. Catal. Today, v.158, p. 121-129, 2010. 